\title{
A ULX IN NGC 4559: A “MINI-CARTWHEEL" SCENARIO?
}

\author{
R. Soria, ${ }^{1}$ M. S. Cropper, ${ }^{1}$ and M. W. Pakull ${ }^{2}$
}

\begin{abstract}
RESUMEN
Estudiamos el peculiar medio alrededor de una ULX en NGC 4559 (con $L_{\mathrm{x}} \approx 2 \times 10^{40} \mathrm{erg} \mathrm{s}^{-1}$ y $M_{\mathrm{BH}} \gtrsim 50 M=$ ). La fuente de rayos $\mathrm{X}$ se localiza cerca del borde (edad $<30 \mathrm{Myr}$ ) de un complejo grande (diámetro $\approx 700$ pc), joven, er forma de anillo, con formación de estrellas posiblemente inducida por el impacto de una galaxia satélite enana a través del disco exterior rico en gas de NGC 4559. Suponemos que interacciones galácticas (incluyendo la caída de nubes de alta velocidad y satélites sobre el disco galáctico) y el medio de baja metalicidad representan condiciones favorables para la formación de remanentes más masivos que las binarias de rayos $\mathrm{X}$ "estándar" y que los que acretan materia de una compañera masiva que llena el lóbulo de Roche.
\end{abstract}

\section{ABSTRACT}

We have studied the peculiar environment around a ULX in NGC 4559 (with $L_{\mathrm{x}} \approx 2 \times 10^{40} \mathrm{erg} \mathrm{s}^{-1}$ and $M_{\mathrm{BH}} \gtrsim 50 M_{\odot}$ ). The X-ray source is located near the rim of a young (age $<30 \mathrm{Myr}$ ), large (diameter $\approx 700$ pc) ring-like star forming complex possibly triggered by the impact of a dwarf satellite galixy through the gas-rich outer disk of NGC 4559. We speculate that galaxy interactions (including the infall of high-relocity clouds and satellites on a galactic disk) and low-metallicity environments offer favourable conditions for the formation of compact remnants more massive than "standard" X-ray binaries, and accreting from a massive Roche-lobe filling companion. Key Words: GALAXIES: INDIVIDUAL (NGC 4559) - GALAXIES: INTERACTIONS - GALAXIES:
SPIRAL - X-RAYS: GALAXIES

\section{A "CANONICAL" ULX IN NGC 4559}

The late-type spiral NGC $4559(d \approx 10$ Mpc: Sanders 2003) hosts two ultra-luminous X-ray sources (ULXs) with isotropic luminosities $\gtrsim 10^{40}$ $\operatorname{erg~s}^{-1}$ (ROSAT study: Vogler, Pietsch \& Bertoldi 1997; XMM-Newton, Chandra study: Cropper et al. 2004). We focus here on the brighter of the two sources, X7 (using the naming convention of Vogler et al. 1997), which presents some of the "canonical" features of ULXs in star-forming galaxies. It has an isotropic luminosity $L_{x} \approx 2 \times 10^{40} \mathrm{erg} \mathrm{s}^{-1}$ in the $0.3-$ $10 \mathrm{keV}$ band, suggesting a mass $\sim 10^{2} M_{\odot}$ for the accreting object. Its X-ray spectrum is well modelled by a power-law (photon index $\Gamma=2.2$ ) with a "soft-excess" below $0.7 \mathrm{keV}$. If the soft component is modelled as a simple blackbody or multicolor blackbody (standard disk spectrum), we obtain a characteristic temperature $k T_{b b} \approx 0.12 \mathrm{keV}$, similar to what is found in some other bright ULXs. We also detect a feature at $\approx 30 \mathrm{mHz}$ in its power-density spectrum, which may be another indication of a high mass, $\gtrsim 50 M_{\odot}$ (Cropper et al. 2004).

\footnotetext{
${ }^{1}$ Mullard Space Science Laboratory, Lniversity College London

${ }^{2}$ Observatoire de Strasbourg, France.
}

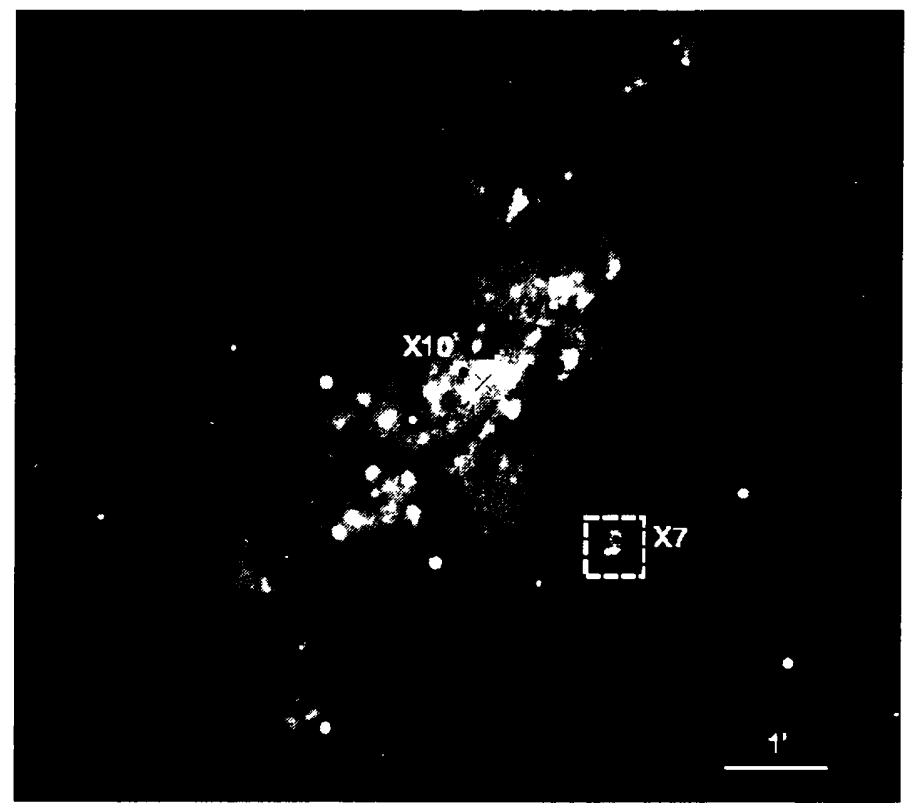

Fig. 1. Both X7 and X10 in the spiral galaxy XC $\mathrm{C}$. 4559 have luminosities $\gtrsim 10^{40} \mathrm{erg} \mathrm{s}^{-1}$ : their location is plotted on a LV image from the Optical Monitor onboard I.MMNewton. The star-forming region around $\mathrm{X} T$ is shown in greater detail in Fig. 2. Here, and in the following inages, North is up, East is to the left. At the distance' of $\mathrm{NGC}+5.59,1^{\prime \prime} \approx .15 \mathrm{pc}$.

\section{A PECULIAR STAR-FORMIIG COMPLEX}

$\mathrm{X} 7$ is located in a large $(d \approx 700 \mathrm{pc})$. isolated star-forming complex, at the outer edse of the stel- 


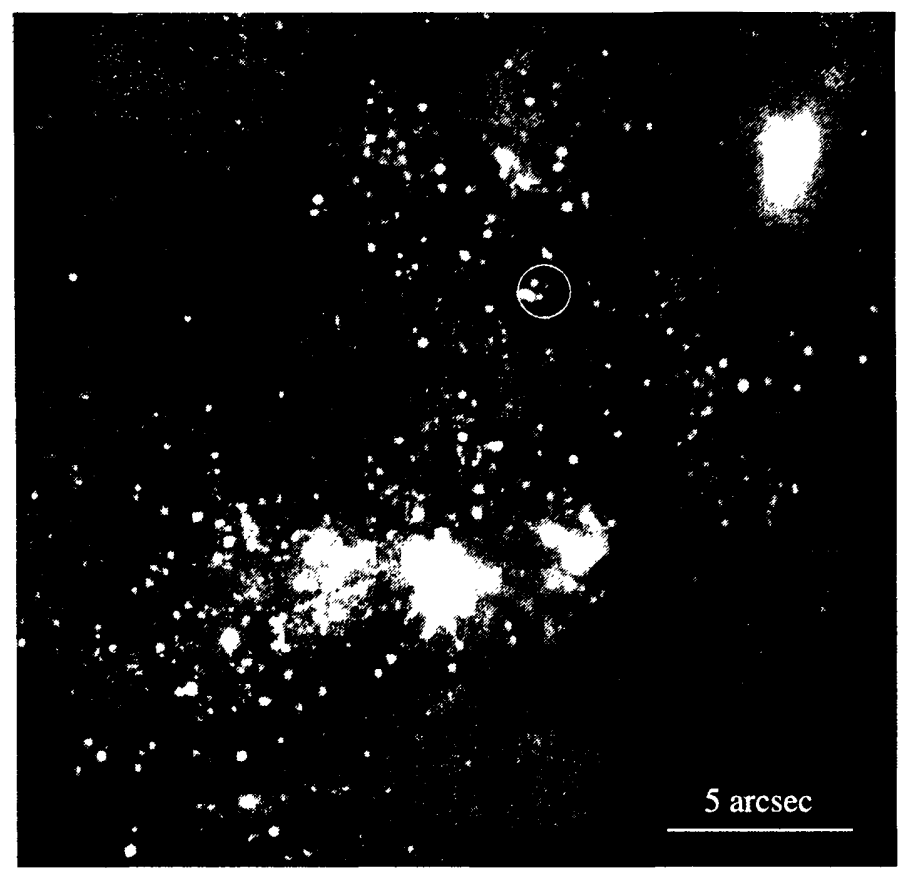

Fig. 2. HST/WFPC2 true-color image of the starforming complex near the X7 ULX. Filters are: $\mathrm{f} 450 \mathrm{w}$ (blue); f555w (green); f814w (red). A likely dIrr satellite galaxy is visilsle near the top right corner. The Chandra error circle for the X-ray source has a radius of 0.7 .

lar disk of NGC 4559 (Fig. 1). From HST/WFPC2 observations, we infer (Soria et al. 2004) an age distribution between 5 and $30 \mathrm{Myr}$ for the local stellar population, dominated by $\mathrm{OB}$ main sequence stars and red supergiants (Fig. 2). Both the optical color distribution and the low blue-to-red supergiant ratio (Soria et al. 2004) are consistent with low (SMCtype) metal abundance (Langer \& Maeder 1995). This is in agreenent with the low metal abundance inferred from our X-ray study (Cropper et al. 2004). A CHFT H $\alpha$ observation (Fig. 3) shows more clearly a shell-like structure for this HII complex, suggesting that an expanding wave of star formation has recently moved from a centre (near but not coincident with the ULX) outwards.

Large, isolated shell- or ring-like star-forming complexes of comparable size (500-1000 pc) and age (10-30 Myr) have been found in other nearby spiral galaxies: eg, in NGC 6946 (eg, Elmegreen, Efremov \& Larsen 2000), and, on a smaller scale, in M83 (Comerón 2001); Gould's Belt in the Milky Way is also similar. We estimate an integrated magnitude $M_{B} \approx-13.5$ for the star-forming complex in NGC 4559, a factor of two brighter than Gould's Belt and four times fainter than the NGC 6946 complex.

Possible explanations for the initial triggering of such star-forming complexes are (Elmegreen et al. 2000): the collapse of a "supergiant molecular

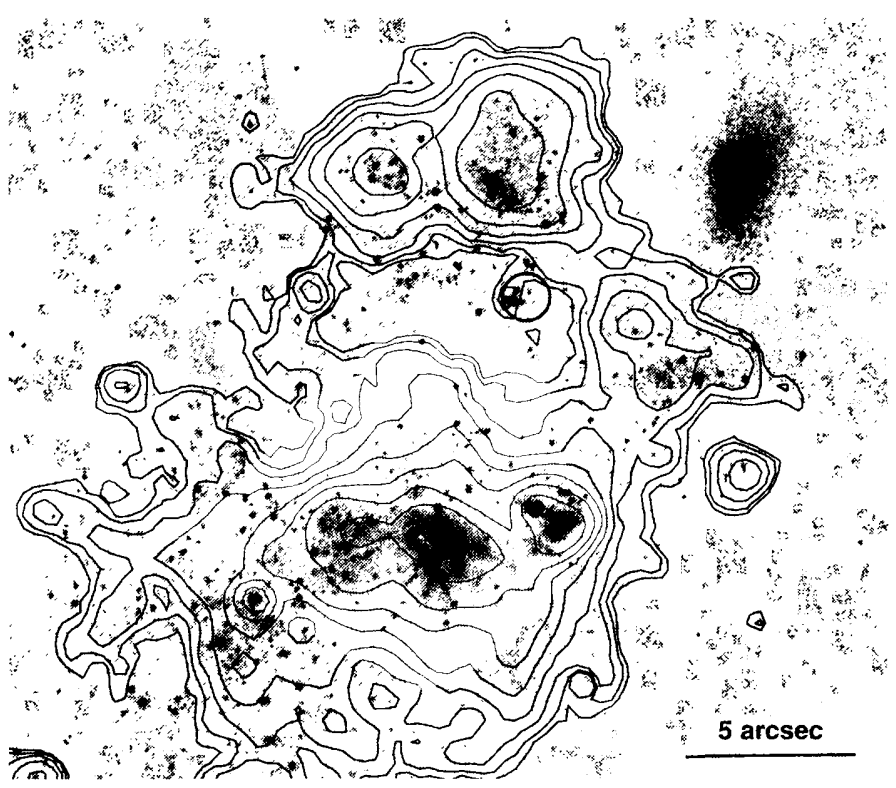

Fig. 3. The ULX is located inside, although not at the center, of a large ring-like HII region. Greyscale image: HST/WFPC2 (PC chip), f450w filter $(\approx B)$. Contours: $\mathrm{H} \alpha$ image from the 4-m CHFT.

cloud" at the end of a spiral arm; a hypernova explosion (which in our case might also have been the progenitor of NGC $4559 \times 7$ ); or the infall of a high-velocity HI cloud or satellite galaxy through the outer galactic disk. In all cases, the initial perturbation creates a radially expanding density wave or ionization front, which sweeps up neutral interstellar medium. Clustered star formation along the expanding bubble rim is triggered by the gravitational collapse of the swept-up material (eg, Elmegreen \& Lada 1977). The large size of the complex in NGC 4559, its location in the outer disk, the lack of other star-forming regions nearby, and the absence of diffuse X-ray emitting gas inside the star-forming complex seem to favour the collision hypothesis over the hypernova model (Tenorio-Tagle et al. 1987).

\section{A DWARF GALAXY PLUNGING THROUGH THE DISK?}

We do indeed see an object that could have plunged through the gas-rich disk of NGC 4559, triggering the expanding density wave: the galaxy located $\approx 7^{\prime \prime}$ north-west of the ULX (Fig. 3). It cannot be a large background elliptical, because its isophotes are too irregular. Its shape, size and luminosity are consistent with a dwarf irregular (or, possibly, a tidally-disturbed dwarf elliptical) at approximately the same distance as NGC 4559. We cannot presently rule out the possibility that it is a chance line-of-sight coincidence, but we suggest that the most natural interpretation is a small satellite galaxy of NGC 4559 . 
If the dIrr galaxy is indeed physically associated with the star-forming complex, its integrated luminosity is $M_{B} \approx-10.7$, with color indices $B-V \approx$ 0.47 and $V-I \approx 0.73$. These colors are typical of a population dominated by $\mathrm{F} 5-\mathrm{F} 8$ main-sequence stars, suggesting an old age. Assuming a single burst of star formation, we infer (using Starburst99, Leitherer et al. 1999) a mass of $\approx 10^{6} M_{\odot}$ for the galaxy and an age $\gtrsim 10^{9} \mathrm{yr}$ for the dominant component of its stellar population. On top of this old component, the galaxy shows two bright clusters with $M_{B} \approx-7.2$ and $M_{B} \approx-.7 .1$; their colors are consistent with an age $\sim 10^{7} \mathrm{yr}$ and masses of $\sim$ a few $\times 10^{3} \mathrm{M}_{\odot}$. Similarly bright star-forming complexes are often found in dIrr galaxies (Parodi \& Binggeli 2003). It is possible that this later episode of star formation in the dIrr satellite may have bcen triggered as this small galaxy passed through the disk of NGC 4559. The projected distance of the dIrr from the centre of the star-forming ring or bubble is $\approx 400 \mathrm{pc}$ (velocity $\approx 30 \mathrm{~km} \mathrm{~s}^{-1}$ over $15 \mathrm{Myr}$ ).

Thus, we could view the star-forming complex in NGC 4559 as a small-scale version of the Cartwheel galaxy, where many young ULXs have been detected in the expanding, star-forming ring (Gao et al. 2003). Apart from the different time and length scales involved, the main difference is that, in the Cartwheel, the initial perturbation causing the expanding density wave is due to the gravitational interaction between the two galaxies; in the case of NGC 4559, it is more likely due to the hydrodynamical interaction between the gas in the satellite galaxy and the gasrich disk.

\section{ENVIRONMENTAL CONDITIONS FAVOURABLE TO ULX FORMATION}

NGC $4559 \times 7$ is a bright $\left(L_{\mathrm{x}}>10^{40} \mathrm{erg} \mathrm{s}^{-1}\right)$ ULX in a low-metallicity environment disturbed by close galaxy interactions. At least one or both of these elements seem to be a common feature for many of the galaxies hosting ULXs (eg, galactic interactions for the Antennae, the Cartwheel, the M81/M82 galaxy group; low metal abundance for the Cartwheel ring, the M81 group dwarfs, I Zw 18). A connection between ULX formation and low-Z environment was suggested in Pakull \& Mirioni (2002).

Assuming that most ULXs can be explained by accreting black holes more massive $\left(\approx 50-100 M_{\odot}\right)$ than those found in nearby X-ray binaries, we speculate that these two environmental conditions may be most favourable for producing massive remnants. Galaxy mergers, tidal interactions, and collisions with satellite galaxies and high-velocity HI clourlfavour clustered star formation. The core of young star clusters may be an environment where massive remnants are formed (through the Spitzer instability, runaway core collapse and merger of the $\mathrm{O}$ stars; eg, Portegies Zwart \& M(cMillan 2002). One of the open questions is what type of cluster (ie. what mass range) offers the best chance for the core collapse/stellar coalescence process to occur within the lifetime of its $O$ stars: superstar clusters $(M \gtrsim$ $\left.10^{5} M_{\odot}\right)$ or smaller clusters $\left(10^{3}<M \lesssim 10^{4} M_{\Xi}\right)$ ?

Low metal abundance implies a lower mass-loss rate in a wind $\left(\dot{M}_{\mathrm{w}} \sim Z^{0.6}\right)$ for the black hole progenitor, leading to a larger core and a more massive remnant. Metallicity also affects the evolution of the donor star (for example. metal-poor stars spend a longer fraction of their life as red supergiants) and the orbital separation of the binary components (which increases for a higher mass-loss rate in a wind. that is, for a higher non-conservative mass transfer). This may affect the timescale in which the donor star fills its Roche lobe (timescale of the ULX phase).

Thanks to H. Jerjen, C. Markwardt, C. Motch. R. Mushotzky, F. Rasio and K. Wu.

\section{REFERENCES}

Comerón, F. 2001, A\&A. 365, 417

Comerón, F., \& Torra, J. 1994, A\&A, 281, 35

Cropper, M. S., Soria, R., Mushotzky, R. F.. Wu. h.. Markwardt, C. B., \& Pakull, M. 2004, MNR.S. in press (astro-ph/0311302)

Elmegrcen, B. G., Efremov, Y N., \& Larsen, S. 2000, ApJ, 535, 748

Elmegreen, B. G., \& Lada, C. J. 1977, ApJ, 214. 7.25

Gao, Y., Wang, Q. D., Appleton, P. V. \& Lucas, R. A. 2003, ApJ, 596, L171

Langer, N., \& Maeder, A. 1995, Ad. A. 295, 68

Leitherer, C., et al. 1999, Ap.JS. 123, 3

Pakull, M. W., \& Mirioni, L. 2002, to appear in the proceedings of the symposium 'New Visions of the Xray Universe', 26-30 November 2001, ESTEC. The Netherlands (astro-ph/0202488)

Parodi, B. R., \& Binggeli, B. 2003, Ad. A. 398. 501

Portegies Zwart, S. F., \& McMillan, S. L. II. 2002. Ip.J. 576,899

Sanders, D. B., Mazzarella, J. M.., Kim, D.-C.. Surace. J. A., \& Soifer, B. T. 2003, AJ, 126, 1607

Soria, R., Cropper, M., Pakull. M.. et al. 2004, MNRAS. in preparation

Tenorio-Tagle, G., Franco, J., Bodenheimer. P.. it Rozyczka, M. 1987, A\&A, 179, 219

Vogler, A., Pietsch, W.. \& Bertoldi. F. 1997. A心. 3. 768 